\title{
Influence of Temperature and Moisture on the Electrical Properties of Leather
}

\author{
Charles E. Weir
}

\begin{abstract}
Apparent, or bulk, dielectric constants and power factors of chrome and vegetable leather and untanned hide are reported for moisture contents up to 30 percent, at temperatures of $23^{\circ} \mathrm{C}, 0^{\circ} \mathrm{C}$, and $-70^{\circ} \mathrm{C}$ over the frequency range 0.75 to 96 kilocycles. Dielectric constants of fibers determined on dry material at 3 kilocycles and $22^{\circ} \mathrm{C}$ are: chrome 5.4; vegetable 6.4; and kangaroo tendon 5.1. Apparent direct-current resistivity decreases from values $>10^{16} \mathrm{ohm}-\mathrm{cm}$ when dry to values $<10^{9} \mathrm{ohm}-\mathrm{cm}$ at 30 -percent moisture. Apparent dielectric constant decreases with frequency and temperature at all moisture contents, the frequency dependence increasing with moisture content. Apparent power factors generally exhibit similar behavior. At $-70^{\circ} \mathrm{C}$ at high moisture contents, a diffuse absorption maximum observed may be due to ice or to permanent dipoles of collagen which are freed by moisture. Observed behavior is interpreted as probably arising from a combination of interfacial polarization at surfaces existing in the fibrous leather and dipolar polarization. Results indicate electrical measurements in this frequency range are probably not reliable for precise quantitative moisture analyses.
\end{abstract}

\section{Introduction}

Adsorption of moisture by hygroscopic materials generally causes a marked change in the electrical properties (conductivity, dielectric constant, and power factor) of such substances. This effect is generally attributed to the high dielectric constant and conductivity of the adsorbed moisture as compared to the much lower values exhibited by the dry adsorbate. In view of the large electrical effects produced by the moisture and the rapidity, relative simplicity, and nondestructive nature of electrical measurements, such determinations have been proposed as methods for quantitative moisture determinations $[3,14,41] .{ }^{1}$ Unfortunately, the materials of particular interest in this connection are usually powders or fibrous materials that possess large adsorption surface areas and, therefore, the results of electrical measurements are not easily interpreted because of the complex physical structure of the specimens. Recently, interest has arisen in application of dielectric measurements for the determination of moisture in leather [27].

This report describes the results of critical studies of the effects of moisture and temperature on the dielectric constant and power factor of leather in the audio and low intermediate frequency range.

In most measurements dealing with a porous material such as leather, two experimental values are recognized, a "real" value associated with the fibers alone, and an "apparent" value that refers to the bulk material including the air in the interstices. To avoid confusion between the former and the real part of the complex dielectric constant, the term "fiber dielectric constant" will be used instead. Apparent values as used are understood to refer to the bulk material.

\footnotetext{
Figures in brackets indicate the literature references at the end of this paper.
}

\section{Experimental Method}

\subsection{Electric Apparatus}

Measurements of capacitance and resistance were obtained with a shielded resistance-capacitance bridge containing a Wagner-earthing circuit. The bridge is shown schematically in figure 1. A detailed discussion of this bridge has been published previously [29]. High-stability input signals in the frequency range 0.75 to $96 \mathrm{kc}$ were provided by a variable frequency oscillator. Bridge balance was ascertained through direct use of earphones at all frequencies as high as $12 \mathrm{kc}$, while at higher frequencies a regenerative, all-wave receiver was used to detect and amplify the output signals.

Fiber dielectric constants were determined by the immersion method $[42,45]$ in a completely shielded, cylindrical, guard-ring condenser. This condenser was constructed of heavy brass, and all surfaces were given a fine machine finish. The guard was located near the top of the condenser below an opening fitted with a reservoir that was used as an overflow. A second opening at the bottom of the condenser was fitted to permit introduction of liquids and suspensions. The capacitance of the empty condenser was $40.22 \mu \mu \mathrm{f}$, a value that was reproducible to within $\pm 0.01 \mu \mu \mathrm{f}$.

All measurements with this condenser were carried out at $22.2^{\circ} \mathrm{C}$ at a frequency of $3 \mathrm{kc}$ in the following manner: The condenser was filled with a suitable solution of ethylene dichloride in benzene. Choice of these liquids resulted from previous studies [26] indicating negligible interaction between leather and liquid. The solution was then circulated through the condenser by using the two openings until a stable and reproducible value of capacitance was obtained. Upon attainment of equilib- 
INPUT

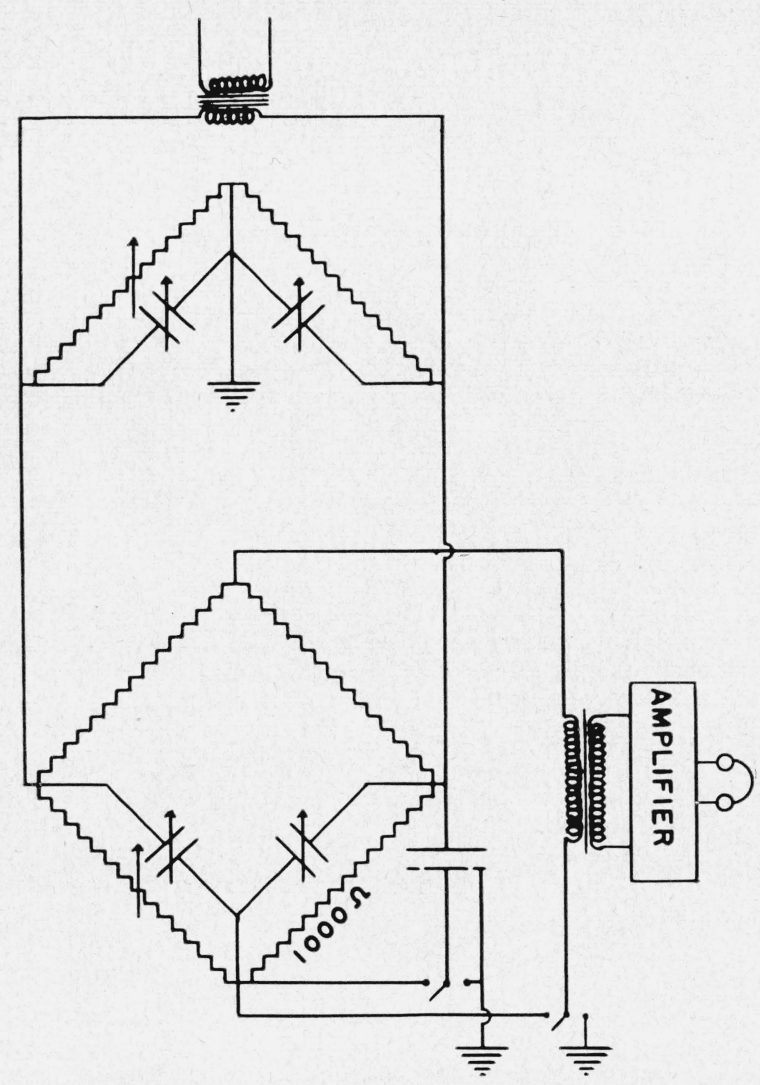

FIGURE 1.-Capacitance-resistance bridge used for measurements of dielectric constant and power factor.

rium, a suspension of finely ground dry leather or collagen was made up by using a portion of the excess liquid, and this suspension was introduced so as to displace the liquid in the condenser. A change in capacitance upon introducing the suspension was indicative of inequality of liquid and solid dielectric constants. The composition of the solution was varied until the dielectric constant of the suspended material was closely bracketed between values for two solutions. The usual method of linear interpolation to determine dielectric constants by the immersion method [11] was difficult to apply with this apparatus as the finely ground solid material used to minimize anisotropic effects tended to settle out or surface slowly and render the suspension nonuniform.

Measurements of apparent dielectric constant were made by using a shielded, parallel-plate, guard-ring condenser. Electrodes were made of heavy brass plates $1 / 2$ in. thick, which were given a fine machine finish. The measuring electrode was $5 \% 16$ in. in diameter, and the normal (empty) plate separation was approximately 0.04 in. The air capacitance was approximately $37 \mu \mu \mathrm{f}$. This condenser was used at all frequencies in the range studied and at temperatures varying between $+20^{\circ}$ to $-70^{\circ} \mathrm{C}$. At lowest frequencies balance was obtained to a precision of $\pm 0.15 \mu \mu \mathrm{f}$ and \pm 0.0002 $\mathrm{obm}$, whereas at highest frequencies balance was obtained to within $\pm 0.01 \mu \mu \mathrm{f}$ and $\pm 0.001 \mathrm{ohm}$.

The condenser was equipped with two sets of three projecting ears spaced at angles of $120^{\circ}$ on its periphery, one set on the top or movable plate, the other directly below on the frame of the condenser. These ears were used to measure the plate spacing, measurements being made to the nearest 0.0001 in. by means of micrometers. Such measurements were made at the conclusion of electrical studies on each specimen. Three set screws mounted in the top plate, equidistant between the ears, permitted resetting the empty condenser to the same spacing measured when the test specimen was in the condenser. The spacings were reproduced to within \pm 0.0002 in. at each ear, and the average of the three settings was reproduced to \pm 0.0001 in. Measurements on the empty condenser under these conditions were used to determine the air capacitance -assumed equal to the vacuum capacitance. In all measurements the top plate of the condenser, which weighed $15 \mathrm{lb}$, was loaded with an additional $20 \mathrm{lb}$ to improve the contact between specimen and plates.

The real part of the dielectric constant, designated as $\epsilon^{\prime}$, was obtained as the ratio of the capacitance of the condenser containing the specimen to that of the empty condenser with the same plate separation. The power factor, designated as cos $\phi$, was obtained from the relationship

$$
\cos \phi=1 / \sqrt{1+\left(R_{p} \omega C_{p}\right)^{2}},
$$

where $R_{p}$ is the equivalent parallel resistance of the condenser containing the specimen, $C_{p}$ it's capacitance, and $\omega$ the angular frequency. The empty condenser exhibited no losses in this frequency range, therefore all losses observed were associated with the test specimens.

\subsection{Test Specimens}

Finely ground specimens of kangaroo tail tendon, chrome hydraulic-packing leather, and vegetable crust leather were used in suspension studies. All specimens were degreased and washed after an initial grinding and then reground. Specimens were dried by exhaustive evacuation at a pressure of less than $1 \mathrm{~mm}$ of mercury for at least $72 \mathrm{hr}$.

Disks $6 \frac{1 / 4}{\mathrm{in}}$. in diameter of chrome hydraulicpacking leather, vegetable crust leather, and unhaired cowhide were split to a uniform thickness of approximately $4 \pm 0.02 \mathrm{~mm}$ by removing portions of grain and flesh layers on a band-knife splitting machine. The disks were degreased, washed, and dried exhaustively in vacuum.

Initial measurements were made on dry specimens, which were then permitted to adsorb moisture from the air to obtain moisture contents as high as approximately 15 percent. For higher moisture contents specimens were exposed to vapors existing above suitable saturated salt solutions or water. Moisture contents below approximately 10 percent 
were obtained by short (a few minutes to as much as $1 \mathrm{hr}$ ) exposures to conditioned air. Uniform moisture distribution was unlikely under these circumstances, but no appreciable change in capacitance or resistance that might be attributed to redistribution of adsorbed moisture was noted during measurements that required as long a period as $48 \mathrm{hr}$. Above 10 percent of moisture, exposures to conditioned atmospheres were conducted for at least $18 \mathrm{hr}$. Despite the longer exposures, appreciable variations in both capacitance and resistance were noted in periods of less than $1 \mathrm{hr}$. Moisture contents were obtained by weighing specimens in a sealed container made of polyethylene film and are based on the dry weight of the specimen. At low moisture contents specimens gained and at high moisture contents lost moisture during introduction and removal from the condenser. Therefore, weights were obtained before and after study, and the average of the two weights was taken to represent the moisture content during measurement. In most instances change in weight noted corresponded to less than 0.1 percent of moisture.

\subsection{Effect of Temperature}

All measurements below room temperature were made in a double-walled insulated container. The parallel-plate condenser and its external loading weight were placed in the innermost container, which was then covered. An ice-water bath, or a dry ice-acetone bath was placed in the outer container to obtain the desired low temperature. Temperatures were measured with a copper-constantan thermocouple mounted inside the condenser. The reference junction of this thermocouple was maintained at $0^{\circ} \mathrm{C}$. Measurements on specimens were generally made after exposure to the desired temperature for $6 \mathrm{hr}$, and although thermal equilibrium was usually not attained in this time, the change in temperature during the time required for measurements was negligible.

\section{Results}

\subsection{Dielectric Constants of Dry Leather and Collagen Fibers}

The results of measurements of dielectric constants of leather and collagen fibers by the immersion method are given in table 1.

Duplicate measurements on each specimen are designated as run 1 and run 2. Following run 1,

TABLE 1. Dielectric constants of dry collagen and leather fibers Temperature $22.25^{\circ} \mathrm{C}$, frequency $3.0 \mathrm{ke}$

\begin{tabular}{|c|c|c|c|}
\hline \multirow{2}{*}{ Material } & \multicolumn{3}{|c|}{$\epsilon^{\prime}$} \\
\hline & Run 1 & Run 2 & Avg \\
\hline $\begin{array}{l}\text { Kangaroo tendon collagen } \\
\text { Hydraulic chrome leather } \\
\text { Vegetable leather }\end{array}$ & $\begin{array}{l}5.12 \\
5.37 \\
6.20\end{array}$ & $\begin{array}{l}5.12 \\
5.41 \\
6.30\end{array}$ & $\begin{array}{l}5.12 \\
5.39 \\
6.25\end{array}$ \\
\hline
\end{tabular}

specimens were exposed to conditioned air at $72^{\circ} \mathrm{F}$ and 50-percent relative humidity for 14 days, then redried in vacuum and the dielectric constants redetermined. Data obtained on the second measurement are referred to as run 2. The agreement between these duplicates indicates that the drying technique employed is reasonably reproducible or that very low moisture contents have little effect on the dielectric properties. The data in table 1 were obtained by bracketing the unknown dielectric constants between solutions differing by not more than 0.15 unit in $\epsilon^{\prime}$. The values given are considered to be accurate to within \pm 0.05 unit in $\epsilon^{\prime}$.

The value for collagen is about the same as the value reported by Errera and Sack [11] on wool-5.4 at $8 \mathrm{kc}$-as well as in general accord with data of Kremen [26] $\left(\epsilon^{\prime}=4.2\right.$ for untanned hide, and $\epsilon^{\prime}=4.6$ for chrome leather at $11 \mathrm{Mc}$ ). It is to be noted that chrome leather exhibits a value of $\epsilon^{\prime}$ slightly higher than that of collagen, but considerably lower than vegetable leather. These data agree with the order reported by Kremen on chrome leather and hide. The values reported here and by Kremen are considerably larger than those calculated from the indices of refraction at optical frequencies of approximately 1.5 as observed by Marriot [30] and Newman [37]. This fact was also mentioned by Errera and Sack [11] in connection with nylon, silk, and wool.

\subsection{Apparent Dielectric Constant and Power Factor}

\section{a. Effect of Moisture}

Measurements on chrome, vegetable, and untanned leather disks of various moisture contents are given in tables 2,3 , and 4 , while a portion of the data on chrome leather is shown in figures 2 and 3.

The following behavior is noted in the data of tables 2,3 , and $4: \epsilon^{\prime}$ increases with increasing moisture content at all frequencies; $\epsilon^{\prime}$ decreases with increasing frequency at all moisture contents, the frequency dependence being least for dry material and greatest for moisture contents of 15 percent or more; $\cos \phi$ increases with increased moisture content; $\cos \phi$ appears to be relatively frequency independent until high moisture levels are reached, that is, 15 percent or more; $\cos \phi$ decreases strongly with increasing frequency at higher moisture levels.

For moisture contents below approximately 15 percent, all measured capacitances and resistances appeared to be independent of time, being reproducible to within 0.1 percent after several hours. At moisture contents greater than 15 percent, definite time dependence was noted, the effect being an increased capacitance and in most instances a decreased power factor at a constant frequency with increasing time. Such effects caused no appreciable error in the measured values, because the changes occurring during the hour required for measurement were generally much less than 1 percent.

\section{b. Direct-Current Resistance}

To ascertain the resistivity of the test specimens and the magnitude of corrections to the effective a-c 


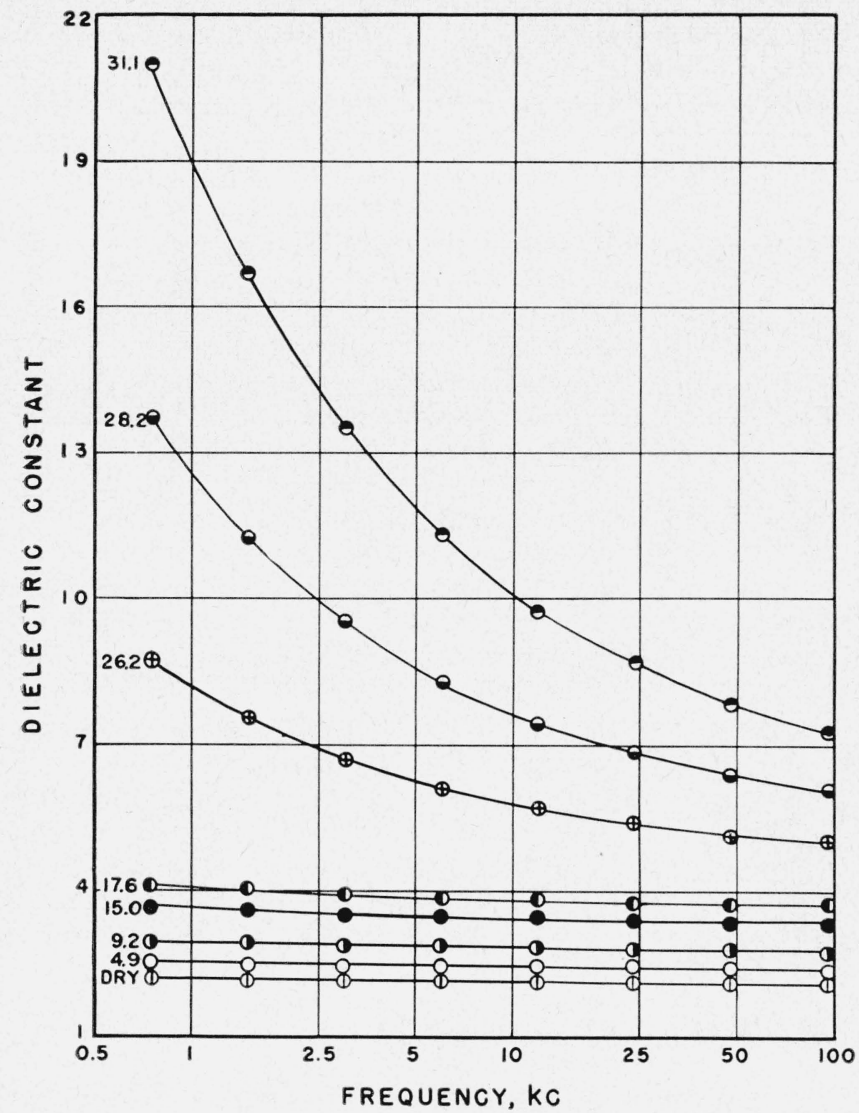

FIgURE 2. Effect of moisture on dielectric constant of chrome leather at $22.3^{\circ} \mathrm{C}$.

๑, 31.1 percent $\mathrm{H}_{2} \mathrm{O} ; \ominus, 28.2$ percent $\mathrm{H}_{2} \mathrm{O} ; \oplus, 26.2$ percent $\mathrm{H}_{2} \mathrm{O} ; \boldsymbol{D}, 17.6$ percent

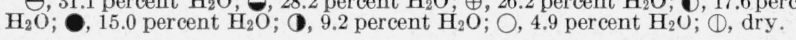

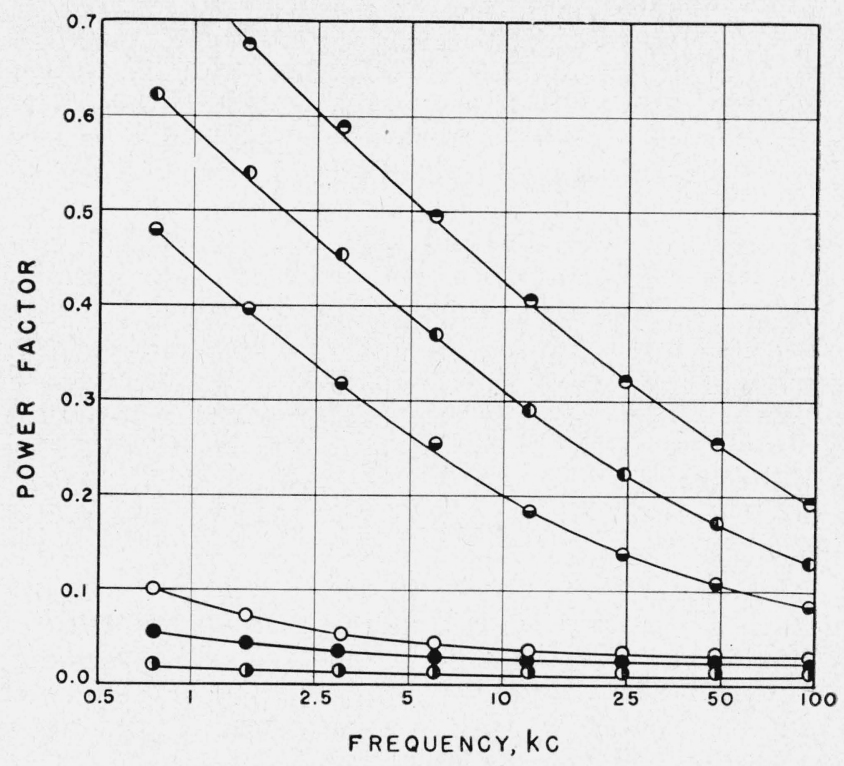

Figure 3. Effect of moisture on power factor of chrome leather at $22.3^{\circ} \mathrm{C}$.

๑, 31.1 percent $\mathrm{H}_{2} \mathrm{O} ; \boldsymbol{\top}, 28.2$ percent $\mathrm{H}_{2} \mathrm{O} ; \ominus$, 26.2 percent $\mathrm{H}_{2} \mathrm{O} ; \bigcirc, 17.6$ per cent $\mathrm{H}_{2} \mathrm{O} ; \mathbf{O}, 15.0$ percent $\mathrm{H}_{2} \mathrm{O} ; \mathbf{O}$, dry.

resistance of the condenser containing the specimen, d-c resistance measurements were carried out on chrome and vegetable leathers immediately following the dielectric measurements. A commercially available megohm bridge containing a guard circuit was used in these studies, all measurements being conducted at room temperature $\left(20^{\circ}\right.$ to $\left.25^{\circ} \mathrm{C}\right)$ with an applied voltage of $500 \mathrm{vdc}$. The results of these measurements, calculated in terms of resistivities, are shown graphically in figure 4.

TABLE 2. Apparent dielectric constant and power factor of hydraulic-packing chrome-tanned leather at $22.25^{\circ} C$

\begin{tabular}{|c|c|c|c|c|c|c|c|c|c|c|c|c|}
\hline \multirow{2}{*}{ Frequency } & \multicolumn{2}{|c|}{ Dry } & \multicolumn{2}{|c|}{$2.63 \% \mathrm{H}_{2} \mathrm{O}$} & \multicolumn{2}{|c|}{$4.91 \% \mathrm{H}_{2} \mathrm{O}$} & \multicolumn{2}{|c|}{$7.28 \% \mathrm{H}_{2} \mathrm{O}$} & \multicolumn{2}{|c|}{$9.23 \% \mathrm{H}_{2} \mathrm{O}$} & \multicolumn{2}{|c|}{$15.03 \% \mathrm{H}_{2} \mathrm{O}$} \\
\hline & $\epsilon^{\prime}$ & $\cos \phi$ & $\epsilon^{\prime}$ & $\cos \phi$ & $\epsilon^{\prime}$ & $\cos \phi$ & $\epsilon^{\prime}$ & $\cos \phi$ & $\epsilon^{\prime}$ & $\cos \phi$ & $\epsilon^{\prime}$ & $\cos \phi$ \\
\hline \multirow[t]{3}{*}{$\begin{array}{l}k c \\
0.75 \\
1.5 \\
3.0 \\
6.0 \\
12.0 \\
24.0 \\
48.0 \\
96.0\end{array}$} & $\begin{array}{l}2.19 \\
2.18 \\
2.16 \\
2.15 \\
2.14 \\
2.13 \\
2.11 \\
2.10\end{array}$ & $\begin{array}{r}0.0182 \\
.0136 \\
.0136 \\
.0140 \\
.0139 \\
.0140 \\
.0138 \\
.0132\end{array}$ & $\begin{array}{l}2.46 \\
2.45 \\
2.44 \\
2.42 \\
2.40 \\
2.38 \\
2.36 \\
2.34\end{array}$ & $\begin{array}{r}0.0110 \\
.0153 \\
.0153 \\
.0160 \\
.0173 \\
.0179 \\
.0187 \\
.0185\end{array}$ & $\begin{array}{l}2.50 \\
2.49 \\
2.48 \\
2.47 \\
2.45 \\
2.43 \\
2.41 \\
2.39\end{array}$ & $\begin{array}{r}0.0154 \\
.0147 \\
.0148 \\
.0157 \\
.0167 \\
.0179 \\
.0191 \\
.0196\end{array}$ & $\begin{array}{l}2.74 \\
2.72 \\
2.70 \\
2.68 \\
2.66 \\
2.64 \\
2.62 \\
2.60\end{array}$ & $\begin{array}{r}0.0163 \\
.0174 \\
.0171 \\
.0173 \\
.0166 \\
.0187 \\
.0202 \\
.0208\end{array}$ & $\begin{array}{l}2.94 \\
2.92 \\
2.89 \\
2.87 \\
2.84 \\
2.82 \\
2.80 \\
2.77\end{array}$ & $\begin{array}{r}0.0226 \\
.0185 \\
.0181 \\
.0177 \\
.0185 \\
.0191 \\
.0203 \\
.0212\end{array}$ & $\begin{array}{l}3.63 \\
3.59 \\
3.54 \\
3.50 \\
3.46 \\
3.43 \\
3.39 \\
3.35\end{array}$ & $\begin{array}{r}0.0546 \\
.0404 \\
.0329 \\
.0289 \\
.0265 \\
.0250 \\
.0252 \\
.0251\end{array}$ \\
\hline & \multicolumn{2}{|c|}{$17.56 \% \mathrm{H}_{2} \mathrm{O}$} & \multicolumn{3}{|c|}{$26.2 \% \mathrm{H}_{2} \mathrm{O}$} & \multicolumn{2}{|c|}{$28.2 \% \mathrm{H}_{2} \mathrm{O}$} & \multicolumn{3}{|c|}{$31.1 \% \mathrm{H}_{2} \mathrm{O}$} & \multicolumn{2}{|c|}{$17.65 \% \mathrm{H}_{2} \mathrm{O}$} \\
\hline & $\epsilon^{\prime}$ & $\cos \phi$ & & $\epsilon^{\prime}$ & $\cos \phi$ & $\epsilon^{\prime}$ & $\cos \phi$ & $\epsilon^{\prime}$ & & $\cos \phi$ & $\epsilon^{\prime}$ & $\cos \phi$ \\
\hline $\begin{array}{r}0.75 \\
1.5 \\
3.0 \\
6.0 \\
12.0 \\
24.0 \\
48.0 \\
96.0\end{array}$ & $\begin{array}{l}4.11 \\
4.01 \\
3.94 \\
3.87 \\
3.82 \\
3.77 \\
3.72 \\
3.68\end{array}$ & $\begin{array}{r}0.0990 \\
.0714 \\
.0538 \\
.0429 \\
.0361 \\
.0322 \\
.0305 \\
.0293\end{array}$ & & $\begin{array}{l}8.75 \\
7.56 \\
6.69 \\
6.11 \\
5.71 \\
5.41 \\
5.19 \\
5.03\end{array}$ & $\begin{array}{r}0.479 \\
.397 \\
.319 \\
.255 \\
.183 \\
.138 \\
.107 \\
.085\end{array}$ & $\begin{array}{r}13.74 \\
11.25 \\
9.52 \\
8.30 \\
7.44 \\
6.84 \\
6.41 \\
6.11\end{array}$ & $\begin{array}{r}0.621 \\
.540 \\
.455 \\
.368 \\
.289 \\
.221 \\
.171 \\
.130\end{array}$ & $\begin{array}{r}21.00 \\
16.69 \\
13.52 \\
11.33 \\
9.77 \\
8.64 \\
7.84 \\
7.30\end{array}$ & & $\begin{array}{r}0.743 \\
.675 \\
.589 \\
.495 \\
.407 \\
.323 \\
.254 \\
.193\end{array}$ & $\begin{array}{l}4.60 \\
4.44 \\
4.34 \\
4.25 \\
4.18 \\
4.11 \\
4.06 \\
4.01\end{array}$ & $\begin{array}{r}0.1270 \\
.0918 \\
.0650 \\
.0529 \\
.0431 \\
.0365 \\
.0338 \\
.0305\end{array}$ \\
\hline
\end{tabular}


TABLE 3. Apparent dielectric constant and power factor of vegetable tanned crust leather at 22.3 $\mathrm{C}$

\begin{tabular}{|c|c|c|c|c|c|c|c|c|c|c|c|c|}
\hline \multirow{2}{*}{ Frequency } & \multicolumn{2}{|c|}{ Dry } & \multicolumn{2}{|c|}{$2.32 \% \mathrm{H}_{2} \mathrm{O}$} & \multicolumn{2}{|c|}{$4.31 \% \mathrm{H}_{2} \mathrm{O}$} & \multicolumn{2}{|c|}{$6.85 \% \mathrm{H}_{2} \mathrm{O}$} & \multicolumn{2}{|c|}{$9.98 \% \mathrm{H}_{2} \mathrm{O}$} & \multicolumn{2}{|c|}{$12.17 \% \mathrm{H}_{2} \mathrm{O}$} \\
\hline & $\epsilon^{\prime}$ & $\cos \phi$ & $\epsilon^{\prime}$ & $\cos \phi$ & $\epsilon^{\prime}$ & $\cos \phi$ & $\epsilon^{\prime}$ & $\cos \phi$ & $\epsilon^{\prime}$ & $\cos \phi$ & $\epsilon^{\prime}$ & $\cos \phi$ \\
\hline \multirow[t]{3}{*}{$\begin{array}{l}\quad k c \\
0.75 \\
1.5 \\
3.0 \\
6.0 \\
12.0 \\
24.0 \\
48.0 \\
96.0\end{array}$} & $\begin{array}{l}2.12 \\
2.12 \\
2.11 \\
2.10 \\
2.09 \\
2.07 \\
2.06 \\
2.05\end{array}$ & $\begin{array}{r}0.0095 \\
.0151 \\
.0142 \\
.0144 \\
.0141 \\
.0137 \\
.0132 \\
.0124\end{array}$ & $\begin{array}{l}2.26 \\
2.25 \\
2.24 \\
2.23 \\
2.22 \\
2.20 \\
2.19 \\
2.18\end{array}$ & $\begin{array}{r}0.0204 \\
.0150 \\
.0161 \\
.0160 \\
.0164 \\
.0168 \\
.0173 \\
.0169\end{array}$ & $\begin{array}{l}2.45 \\
2.43 \\
2.42 \\
2.41 \\
2.39 \\
2.37 \\
2.36 \\
2.34\end{array}$ & $\begin{array}{r}0.0153 \\
.0183 \\
.0169 \\
.0168 \\
.0175 \\
.0182 \\
.0191 \\
.0192\end{array}$ & $\begin{array}{l}2.87 \\
2.84 \\
2.82 \\
2.80 \\
2.79 \\
2.76 \\
2.74 \\
2.74\end{array}$ & $\begin{array}{r}0.0309 \\
.0231 \\
.0210 \\
.0204 \\
.0201 \\
.0204 \\
.0215 \\
.0220\end{array}$ & $\begin{array}{l}3.61 \\
3.56 \\
3.52 \\
3.48 \\
3.44 \\
3.41 \\
3.37 \\
3.34\end{array}$ & $\begin{array}{r}0.0526 \\
.0421 \\
.0335 \\
.0291 \\
.0266 \\
.0246 \\
.0246 \\
.0236\end{array}$ & $\begin{array}{l}3.84 \\
3.75 \\
3.67 \\
3.62 \\
3.56 \\
3.52 \\
3.48 \\
3.47\end{array}$ & $\begin{array}{r}0.0972 \\
.0763 \\
.0538 \\
.0420 \\
.0346 \\
.0297 \\
.0277 \\
.0253\end{array}$ \\
\hline & \multicolumn{2}{|c|}{$13.67 \% \mathrm{H}_{2} \mathrm{O}$} & \multicolumn{2}{|c|}{$15.3 \% \mathrm{H}_{2} \mathrm{O}$} & \multicolumn{2}{|c|}{$17.8 \% \mathrm{H}_{2} \mathrm{O}$} & \multicolumn{2}{|c|}{$22.7 \% \mathrm{H}_{2} \mathrm{O}$} & \multicolumn{2}{|c|}{$25.5 \% \mathrm{H}_{2} \mathrm{O}$} & \multicolumn{2}{|c|}{$27.4 \% \mathrm{H}_{2} \mathrm{O}$} \\
\hline & $\epsilon^{\prime}$ & $\cos \phi$ & $\epsilon^{\prime}$ & $\cos \phi$ & $\epsilon^{\prime}$ & $\cos \phi$ & $\epsilon^{\prime}$ & $\cos \phi$ & $\epsilon^{\prime}$ & $\cos \phi$ & $\epsilon^{\prime}$ & $\cos \phi$ \\
\hline $\begin{array}{l}0.75 \\
1.5 \\
3.0 \\
6.0 \\
12.0 \\
24.0 \\
48.0 \\
96.0\end{array}$ & $\begin{array}{l}4.19 \\
4.01 \\
3.90 \\
3.81 \\
3.75 \\
3.70 \\
3.64 \\
3.60\end{array}$ & $\begin{array}{r}0.1432 \\
.1042 \\
.0772 \\
.0575 \\
.0449 \\
.0369 \\
.0325 \\
.0301\end{array}$ & $\begin{array}{l}4.82 \\
4.51 \\
4.30 \\
4.15 \\
4.05 \\
3.99 \\
3.91 \\
3.86\end{array}$ & $\begin{array}{r}0.2200 \\
.1642 \\
.1190 \\
.0870 \\
.0658 \\
.0505 \\
.0415 \\
.0343\end{array}$ & $\begin{array}{l}\text { 6. } 31 \\
5.61 \\
5.09 \\
4.84 \\
4.63 \\
4.49 \\
4.38 \\
4.31\end{array}$ & $\begin{array}{l}0.329 \\
.266 \\
.211 \\
.154 \\
.113 \\
.0824 \\
.0626 \\
.0478\end{array}$ & $\begin{array}{r}10.36 \\
9.46 \\
7.95 \\
6.83 \\
6.21 \\
5.64 \\
5.34 \\
5.14\end{array}$ & $\begin{array}{r}0.396 \\
.392 \\
.366 \\
.318 \\
.255 \\
.191 \\
.141 \\
.0996\end{array}$ & $\begin{array}{r}14.35 \\
12.80 \\
10.88 \\
9.00 \\
7.65 \\
6.75 \\
6.15 \\
5.80\end{array}$ & $\begin{array}{r}0.480 \\
.422 \\
.407 \\
.390 \\
.344 \\
.280 \\
.216 \\
.153\end{array}$ & $\begin{array}{r}15.40 \\
14.12 \\
12.62 \\
10.78 \\
9.03 \\
7.69 \\
6.76 \\
6.22\end{array}$ & $\begin{array}{r}0.602 \\
.474 \\
.412 \\
.398 \\
.379 \\
.331 \\
.272 \\
.200\end{array}$ \\
\hline
\end{tabular}

TABLE 4. Apparent dielectric constant and power factor of untanned hide at $22.25^{\circ} \mathrm{C}$

\begin{tabular}{|c|c|c|c|c|c|c|c|c|c|c|}
\hline \multirow{2}{*}{ Frequency } & \multicolumn{2}{|c|}{ Dry } & \multicolumn{2}{|c|}{$2.83 \% \mathrm{H}_{2} \mathrm{O}$} & \multicolumn{2}{|c|}{$5.64 \% \mathrm{H}_{2} \mathrm{O}$} & \multicolumn{2}{|c|}{$8.52 \% \mathrm{H}_{2} \mathrm{O}$} & \multicolumn{2}{|c|}{$11.3 \% \mathrm{H}_{2} \mathrm{O}$} \\
\hline & $\epsilon^{\prime}$ & $\cos \phi$ & $\epsilon^{\prime}$ & $\cos \phi$ & $\epsilon^{\prime}$ & $\cos \phi$ & $\epsilon^{\prime}$ & $\cos \phi$ & $\epsilon^{\prime}$ & $\cos \phi$ \\
\hline \multirow[t]{3}{*}{$\begin{array}{l}k c \\
0.75 \\
1.5 \\
3.0 \\
6.0 \\
12.0 \\
24.0 \\
48.0 \\
96.0\end{array}$} & $\begin{array}{l}1.91 \\
1.90 \\
1.88 \\
1.87 \\
1.86 \\
1.85 \\
1.84 \\
1.83\end{array}$ & $\begin{array}{r}0.0132 \\
.0135 \\
.0129 \\
.0132 \\
.0124 \\
.0117 \\
.0112 \\
.0101\end{array}$ & $\begin{array}{l}2.04 \\
2.03 \\
2.02 \\
2.00 \\
1.99 \\
1.97 \\
1.96 \\
1.94\end{array}$ & $\begin{array}{r}0.0119 \\
.0153 \\
.0159 \\
.0161 \\
.0164 \\
.0168 \\
.0173 \\
.0163\end{array}$ & $\begin{array}{l}2.23 \\
2.22 \\
2.21 \\
2.19 \\
2.18 \\
2.15 \\
2.13 \\
2.12\end{array}$ & $\begin{array}{r}0.0182 \\
.0180 \\
.0180 \\
.0182 \\
.0190 \\
.0196 \\
.0208 \\
.0206\end{array}$ & $\begin{array}{l}2.47 \\
2.46 \\
2.44 \\
2.42 \\
2.40 \\
2.37 \\
2.35 \\
2.33\end{array}$ & $\begin{array}{r}0.0205 \\
.0224 \\
.0202 \\
.0206 \\
.0212 \\
.0219 \\
.0234 \\
.0232\end{array}$ & $\begin{array}{l}2.79 \\
2.76 \\
2.73 \\
2.71 \\
2.68 \\
2.65 \\
2.60 \\
2.58\end{array}$ & $\begin{array}{r}0.0256 \\
.0251 \\
.0241 \\
.0238 \\
.0249 \\
.0253 \\
.0267 \\
.0248\end{array}$ \\
\hline & \multicolumn{2}{|c|}{$14.9 \% \mathrm{H}_{2} \mathrm{O}$} & \multicolumn{3}{|c|}{$19.7 \% \mathrm{H}_{2} \mathrm{O}$} & \multicolumn{2}{|c|}{$31.7 \% \mathrm{H}_{2} \mathrm{O}$} & \multicolumn{3}{|c|}{$40.3 \% \mathrm{H}_{2} \mathrm{O}$} \\
\hline & $\epsilon^{\prime}$ & $\cos \phi$ & & & $\cos \phi$ & $\epsilon^{\prime}$ & $\cos \phi$ & & $\epsilon^{\prime}$ & $\cos \phi$ \\
\hline $\begin{array}{l}0.75 \\
1.5 \\
3.0 \\
6.0 \\
12.0 \\
24.0 \\
48.0 \\
96.0\end{array}$ & $\begin{array}{l}3.27 \\
3.24 \\
3.22 \\
3.18 \\
3.16 \\
3.12 \\
3.09 \\
3.05\end{array}$ & $\begin{array}{r}0.0261 \\
.0245 \\
.0234 \\
.0236 \\
.0247 \\
.0265 \\
.0265\end{array}$ & & & $\begin{array}{r}0.0757 \\
.0572 \\
.0445 \\
.0380 \\
.0335 \\
.0322 \\
.0328 \\
.0316\end{array}$ & $\begin{array}{r}10.58 \\
10.29 \\
7.80 \\
6.94 \\
6.35 \\
5.95 \\
5.69 \\
5.51\end{array}$ & $\begin{array}{r}0.578 \\
.428 \\
.388 \\
.309 \\
.235 \\
.171 \\
.126 \\
.0911\end{array}$ & & $\begin{array}{r}19.80 \\
16.52 \\
13.81 \\
11.60 \\
10.02 \\
8.86 \\
8.04 \\
7.50\end{array}$ & $\begin{array}{r}0.897 \\
.810 \\
.707 \\
.593 \\
.478 \\
.367 \\
.274 \\
.192\end{array}$ \\
\hline
\end{tabular}

It will be noted that the resistivities are rather high, even at higher moisture contents. The magnitude of these values is such that at room temperature no significant correction for d-c resisistivity is required, since a-c resistivities were invariably much less than the $d-c$ values. It was also observed that the resistance was independent of time at low moisture contents, but at higher moisture levels a time dependent drift of resistance to higher values was noted.

\section{c. Effect of Temperature and Moisture}

To obtain further information on the phenomena responsible for the observed behavior of $\epsilon^{\prime}$ and $\cos \phi$ at room temperature, as well as data on the temperature dependence of these quantities, measurements were made at room temperature and at approximately $0^{\circ} \mathrm{C}$ and $-70^{\circ} \mathrm{C}$. As noted previously, thermal equilibrium was generally not attained at reduced temperatures so that measurements were conducted with a very slowly decreasing temperature, the actual temperature being measured thermoelectrically. Prior to each measurement at a reduced temperature, a set of data was obtained at room temperature, in order to ascertain any hysteresis effect resulting from a previous cooling. In general, measurements were conducted at $0^{\circ} \mathrm{C}$ before cooling to $-70^{\circ} \mathrm{C}$, but in some instances this order was reversed because the experiments indicated that hysteresis effects did not affect the general nature of the results. The results of these experiments are given in tables 5, 6, and 7 , the sets of data obtained at room temperature being given in the columns to the left of the succeeding data obtained at reduced temperatures. 


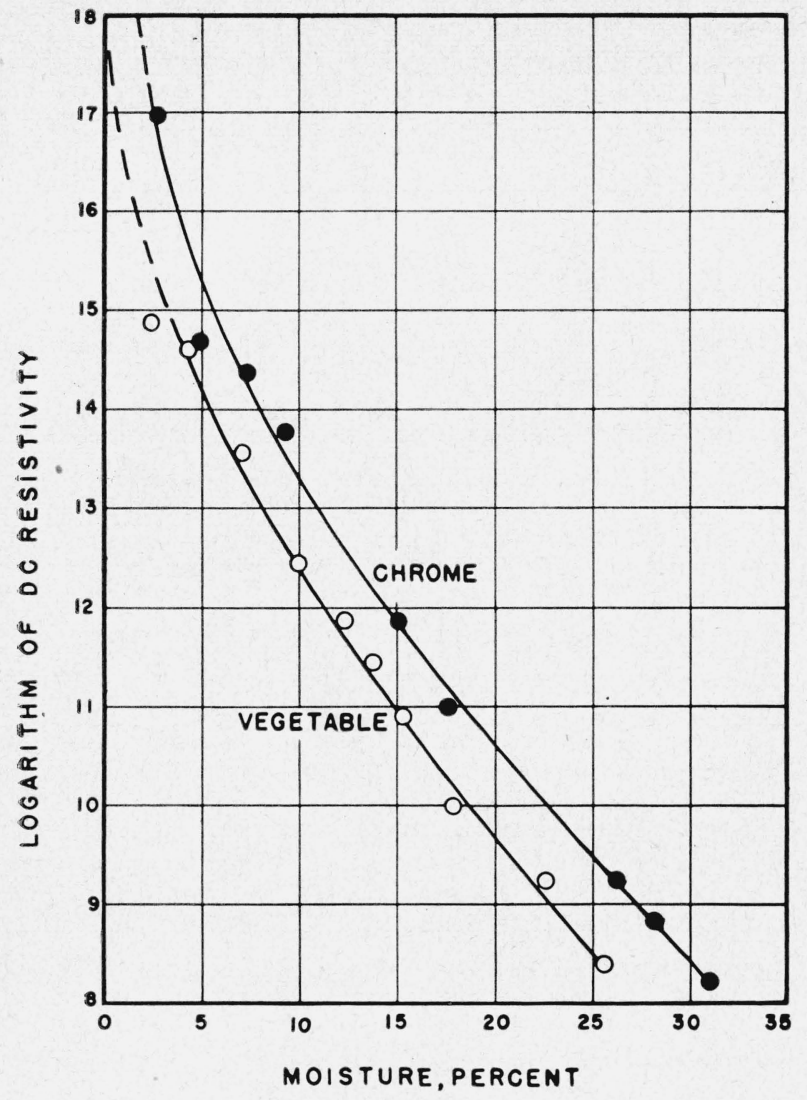

Figure 4. Effect of moisture on $d$-c resistance at $22^{\circ} \mathrm{C}$. , Chrome leather: , vegetable leather.

Apparent dielectric constants shown in these tables were obtained by use of the air capacitance of the condenser as measured at room temperature, since it was not feasible to measure the plate separation while the condenser was subjected to low temperatures. A measure of the error involved in this procedure may be obtained by manipulations of the equation for the capacitance of a parallel plate condenser and use of the coefficients of thermal expansion of brass [17] and leather [47]. For the experimental quantities involved here such calculations indicate an error of 1.4 percent in capacitance for a temperature change of $100 \mathrm{deg} \mathrm{C}$. The corresponding error in $\epsilon^{\prime}$ is also 1.4 percent. As the expansivity of leather in air is probably smaller than the expansivity used in the calculation [47], the probable error is expected to be less than 1.4 percent. Such an error will in no way affect the following conclusions drawn from these data: $\epsilon^{\prime}$ decreases with decreasing temperature at all frequencies and moisture contents, and is strongly temperature dependent at high moisture levels; $\epsilon^{\prime}$ decreases with increasing frequency at all temperatures and moisture contents; $\cos \phi$ generally decreases with decreasing temperature at a fixed frequency for very low and very high moisture contents, being strongly temperature dependent at higher moisture levels.

At intermediate moisture contents $\cos \phi$ may in-

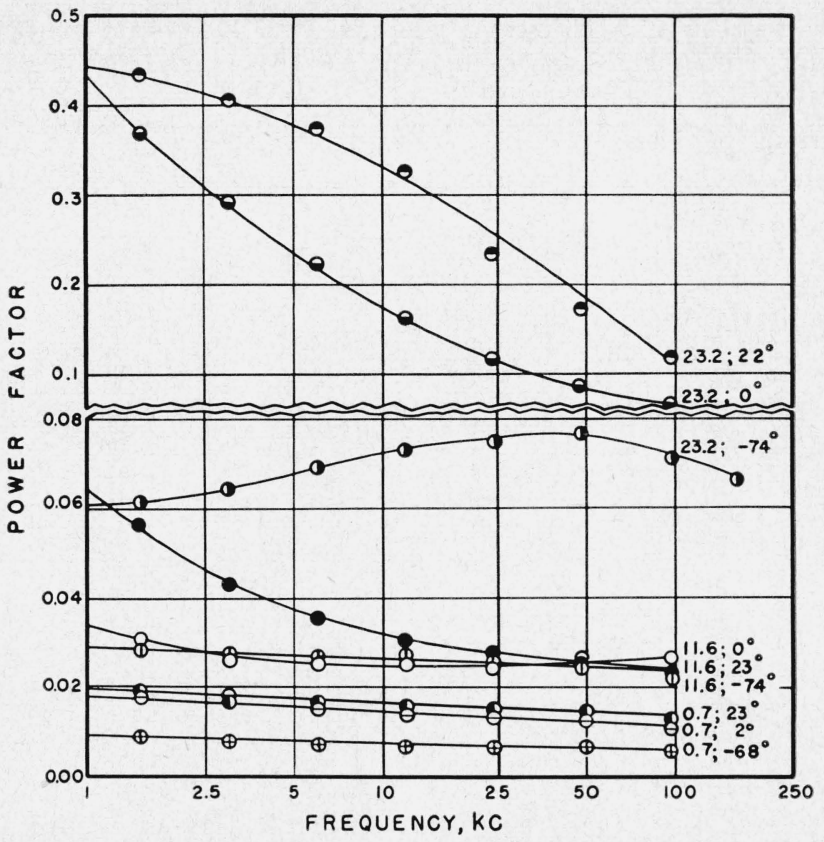

FIGURE 5. Effects of moisture and temperature on dielectric constant of vegetable leather.

23.2 percent $\mathrm{H}_{2} \mathrm{O}: \bullet, 22^{\circ} \mathrm{C} ; \ominus, 0^{\circ} \mathrm{C} ;-74^{\circ} \mathrm{C}, 0.7$ percent $\mathrm{H}_{2} \mathrm{O}: \bullet, 23^{\circ} \mathrm{C}$; $0,2^{\circ} \mathrm{C} ; \mathbf{O},-68^{\circ} \mathrm{C}$

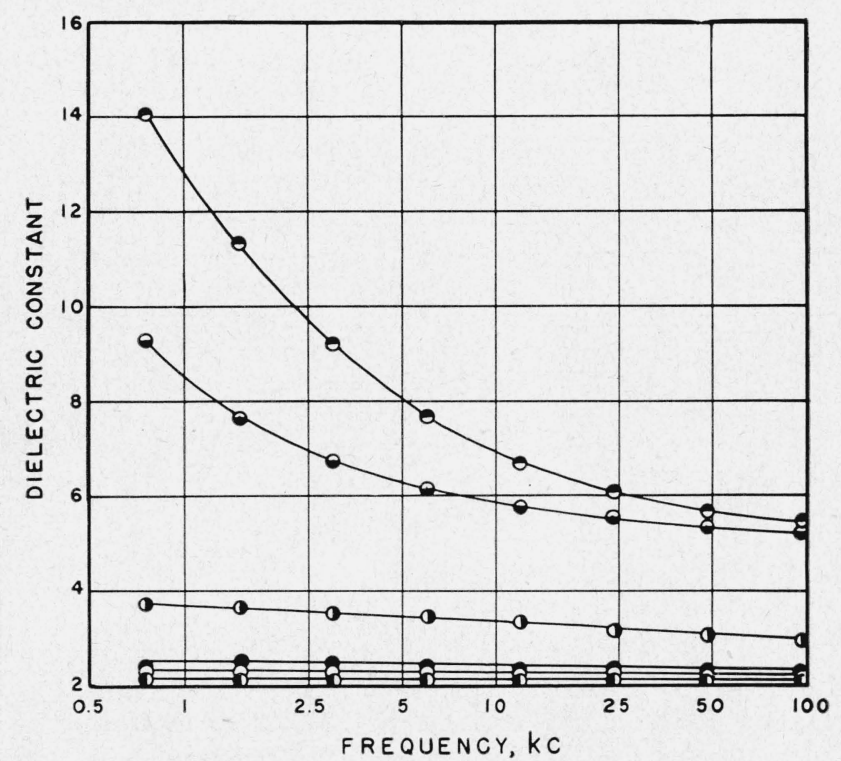

FIGURE 6. Effects of moisture and temperature on power factor of vegetable leather.

23.2 percent $\mathrm{H}_{2} \mathrm{O}: \theta, 22^{\circ} \mathrm{C} ; \ominus, 0^{\circ} \mathrm{C} ; \mathbf{O},-74^{\circ} \mathrm{C}, 11.6$ percent $\mathrm{H}_{2} \mathrm{O}:$ $\mathrm{O}, 0^{\circ} \mathrm{C} ; \oplus,-71^{\circ} \mathrm{C}$. 0.7 percent $\mathrm{H}_{2} \mathrm{O}: \mathbf{O}, 23^{\circ} \mathrm{C} ; \ominus, 2^{\circ} \mathrm{C} ; \oplus,-68^{\circ} \mathrm{C}$.

crease or decrease with decreasing temperature depending on frequency and temperature.

At low temperatures and high moisture contents $\cos \phi$ (as well as $\epsilon^{\prime \prime}$ ) exhibits a somewhat broadened but unmistakable absorption maximum for hide and vegetable leathers, and such a maximum would appear to be present in chrome leather at higher moisture contents. Representative data are shown in figures 5 and 6 . 
TABLE 5. Effect of temperature and moisture on apparent dielectric constant and power factor of hide collagen

\begin{tabular}{|c|c|c|c|c|c|c|c|c|}
\hline \multirow{3}{*}{$\begin{array}{c}\text { Fre- } \\
\text { quency }\end{array}$} & \multicolumn{8}{|c|}{ Dry hide } \\
\hline & \multicolumn{2}{|c|}{$20.5^{\circ} \mathrm{C}$} & \multicolumn{2}{|c|}{$-70.0^{\circ} \mathrm{C}$} & \multicolumn{2}{|c|}{$22.0^{\circ} \mathrm{C}$} & \multicolumn{2}{|c|}{$2^{\circ} \mathrm{C}$} \\
\hline & $\epsilon^{\prime}$ & $\cos \phi$ & $\epsilon^{\prime}$ & $\cos \phi$ & $\epsilon^{\prime}$ & $\cos \phi$ & $\epsilon^{\prime}$ & $\cos \phi$ \\
\hline $\begin{array}{l}k c \\
0.75 \\
1.5 \\
3.0 \\
6.0 \\
12.0 \\
24.0 \\
48.0 \\
96.0\end{array}$ & $\begin{array}{l}1.88 \\
1.88 \\
1.87 \\
1.86 \\
1.84 \\
1.83 \\
1.82 \\
1.81\end{array}$ & $\begin{array}{c}0.00976 \\
.0155 \\
.0153 \\
.0147 \\
.0140 \\
.0133 \\
.0130 \\
.0113\end{array}$ & $\begin{array}{l}1.74 \\
1.71 \\
1.71 \\
1.70 \\
1.70 \\
1.70 \\
1.69 \\
1.69\end{array}$ & $\begin{array}{r}0.00198 \\
.00365 \\
.00400 \\
.00473 \\
.00474 \\
.00441 \\
.00429 \\
.00377\end{array}$ & $\begin{array}{l}1.88 \\
1.88 \\
1.87 \\
1.86 \\
1.85 \\
1.84 \\
1.83 \\
1.82\end{array}$ & $\begin{array}{c}0.00912 \\
.0137 \\
.0148 \\
.0145 \\
.0140 \\
.0133 \\
.0127 \\
.0113\end{array}$ & $\begin{array}{l}1.84 \\
1.84 \\
1.83 \\
1.82 \\
1.81 \\
1.80 \\
1.79 \\
1.78\end{array}$ & $\begin{array}{r}0.0155 \\
.0113 \\
.0139 \\
.0130 \\
.0123 \\
.0112 \\
.0105 \\
.0093\end{array}$ \\
\hline \multirow{3}{*}{$\begin{array}{l}48.0 \\
96.0\end{array}$} & \multicolumn{8}{|c|}{$16.0 \% \mathrm{H}_{2} \mathrm{O}$} \\
\hline & \multicolumn{2}{|c|}{$22.5^{\circ} \mathrm{C}$} & \multicolumn{2}{|c|}{$-68^{\circ} \mathrm{C}$} & \multicolumn{2}{|c|}{$23.0^{\circ} \mathrm{C}$} & \multicolumn{2}{|c|}{$2^{\circ} \mathrm{C}$} \\
\hline & $\epsilon^{\prime}$ & $\cos \phi$ & $\epsilon^{\prime}$ & $\cos \phi$ & $\epsilon^{\prime}$ & $\cos \phi$ & $\epsilon^{\prime}$ & $\cos \phi$ \\
\hline $\begin{array}{r}0.75 \\
1.5 \\
3.0 \\
6.0 \\
12.0 \\
24.0 \\
48.0 \\
96.0\end{array}$ & $\begin{array}{l}\text { 3. } 27 \\
\text { 3. } 24 \\
\text { 3. } 21 \\
\text { 3. } 18 \\
\text { 3. } 14 \\
\text { 3. } 10 \\
\text { 3. } 07 \\
\text { 3. } 03\end{array}$ & $\begin{array}{r}0.0286 \\
.0263 \\
.0249 \\
.0251 \\
.0252 \\
.0263 \\
.0279 \\
.0277\end{array}$ & $\begin{array}{l}2.42 \\
2.40 \\
2.37 \\
2.33 \\
2.30 \\
2.26 \\
2.22 \\
2.18\end{array}$ & $\begin{array}{r}0.0281 \\
.0259 \\
.0291 \\
.0303 \\
.0319 \\
.0323 \\
.0313 \\
.0326\end{array}$ & $\begin{array}{l}\text { 3. } 22 \\
\text { 3. } 19 \\
\text { 3. } 15 \\
\text { 3. } 12 \\
\text { 3. } 08 \\
3.05 \\
\text { 3. } 01 \\
2.97\end{array}$ & $\begin{array}{r}0.0291 \\
.0286 \\
.0263 \\
.0256 \\
.0249 \\
.0263 \\
.0279 \\
.0279\end{array}$ & $\begin{array}{l}3.03 \\
3.01 \\
2.99 \\
2.95 \\
2.93 \\
2.89 \\
2.86 \\
2.83\end{array}$ & $\begin{array}{r}0.0208 \\
.0243 \\
.0237 \\
.0241 \\
.0255 \\
.0263 \\
.0283 \\
.0282\end{array}$ \\
\hline \multirow{3}{*}{$\begin{array}{l}48.0 \\
96.0\end{array}$} & \multicolumn{8}{|c|}{$37.1 \% \mathrm{H}_{2} \mathrm{O}$} \\
\hline & \multicolumn{2}{|c|}{$22.3^{\circ} \mathrm{C}$} & \multicolumn{2}{|c|}{$-74.7^{\circ} \mathrm{C}$} & \multicolumn{2}{|c|}{$22.1^{\circ} \mathrm{C}$} & \multicolumn{2}{|c|}{$0^{\circ} \mathrm{C}$} \\
\hline & $\epsilon^{\prime}$ & $\cos \phi$ & $\epsilon^{\prime}$ & $\cos \phi$ & $\epsilon^{\prime}$ & $\cos \phi$ & $\epsilon^{\prime}$ & $\cos \phi$ \\
\hline $\begin{array}{r}0.75 \\
1.5 \\
3.0 \\
6.0 \\
12.0 \\
24.0 \\
48.0 \\
96.0\end{array}$ & $\begin{array}{r}19.20 \\
14.96 \\
12.00 \\
9.90 \\
8.54 \\
7.66 \\
7.08 \\
6.74\end{array}$ & $\begin{array}{r}0.773 \\
.701 \\
.614 \\
.520 \\
.412 \\
.305 \\
.221 \\
.153\end{array}$ & $\begin{array}{l}4.07 \\
3.97 \\
3.83 \\
3.69 \\
3.52 \\
3.34 \\
3.15 \\
2.98\end{array}$ & $\begin{array}{r}0.0720 \\
.0735 \\
.0854 \\
.0953 \\
.1078 \\
.1178 \\
.1275 \\
.0617\end{array}$ & $\begin{array}{r}19.60 \\
15.11 \\
12.22 \\
10.20 \\
8.90 \\
8.06 \\
7.50 \\
7.15\end{array}$ & $\begin{array}{r}0.825 \\
.747 \\
.650 \\
.535 \\
.413 \\
.300 \\
.215 \\
.148\end{array}$ & $\begin{array}{r}11.70 \\
9.79 \\
8.58 \\
7.79 \\
7.29 \\
6.95 \\
6.69 \\
6.51\end{array}$ & $\begin{array}{r}0.610 \\
.502 \\
.385 \\
.283 \\
.200 \\
.141 \\
.106 \\
.0805\end{array}$ \\
\hline
\end{tabular}

TABLE 6. Effect of temperature and moisture on apparent dielectric constant and power factor of chrome leather

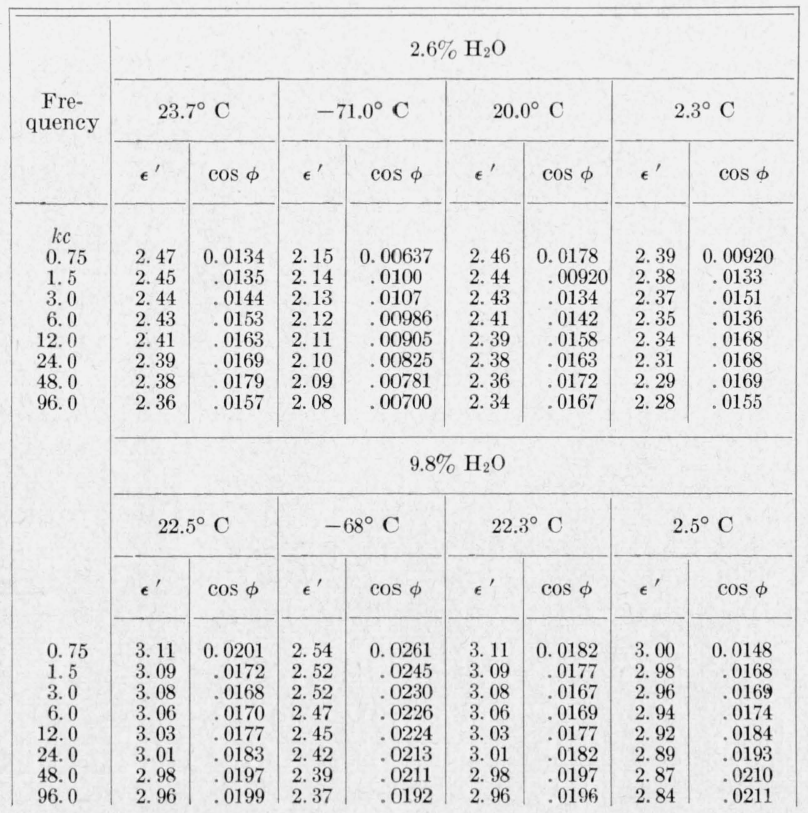

TABLE 6. Effect of temperature and moisture on apparent dielectric constant and power factor of chrome leather-Con.

\begin{tabular}{|c|c|c|c|c|c|c|c|c|}
\hline & \multicolumn{8}{|c|}{$21.5 \% \mathrm{H}_{2} \mathrm{O}$} \\
\hline & \multicolumn{2}{|c|}{$25^{\circ} \mathrm{C}$} & \multicolumn{2}{|c|}{$-65^{\circ} \mathrm{C}$} & \multicolumn{2}{|c|}{$24^{\circ} \mathrm{C}$} & \multicolumn{2}{|c|}{$2^{\circ} \mathrm{C}$} \\
\hline & $\epsilon^{\prime}$ & $\cos \phi$ & $\epsilon^{\prime}$ & $\cos \phi$ & $\epsilon^{\prime}$ & $\cos \phi$ & $\epsilon^{\prime}$ & $\cos \phi$ \\
\hline $\begin{array}{l}0.75 \\
1.5 \\
3.0 \\
6.0 \\
12.0 \\
24.0 \\
48.0 \\
96.0\end{array}$ & $\begin{array}{l}\text { 6. } 53 \\
\text { 5. } 89 \\
\text { 5. } 46 \\
5.16 \\
4.96 \\
4.81 \\
4.69 \\
4.60\end{array}$ & $\begin{array}{c}0.347 \\
.227 \\
.199 \\
.143 \\
.105 \\
.0792 \\
.0640 \\
.0520\end{array}$ & $\begin{array}{l}3.58 \\
3.53 \\
3.48 \\
3.43 \\
3.36 \\
3.30 \\
3.23 \\
3.15\end{array}$ & $\begin{array}{r}0.0309 \\
.0327 \\
.0340 \\
.0368 \\
.0395 \\
.0439 \\
.0500 \\
.0518\end{array}$ & $\begin{array}{l}6.31 \\
5.77 \\
55.42 \\
5.17 \\
5.00 \\
4.86 \\
4.75 \\
4.65\end{array}$ & $\begin{array}{l}0.314 \\
.237 \\
.172 \\
.126 \\
.0945 \\
.0727 \\
.0603 \\
.0500\end{array}$ & $\begin{array}{l}5.05 \\
4.86 \\
4.71 \\
4.63 \\
4.54 \\
4.45 \\
4.37 \\
4.31\end{array}$ & $\begin{array}{l}0.137 \\
.0995 \\
.0741 \\
.0593 \\
.0494 \\
.0429 \\
.0401 \\
.0364\end{array}$ \\
\hline \multirow{3}{*}{96.0} & \multicolumn{8}{|c|}{$30.6 \% \mathrm{H}_{2} \mathrm{O}$} \\
\hline & \multicolumn{2}{|c|}{$23.0^{\circ} \mathrm{C}$} & \multicolumn{2}{|c|}{$-73.0^{\circ} \mathrm{C}$} & \multicolumn{2}{|c|}{$20.0^{\circ} \mathrm{C}$} & \multicolumn{2}{|c|}{$1.0^{\circ} \mathrm{C}$} \\
\hline & $\epsilon^{\prime}$ & $\cos \phi$ & $\epsilon^{\prime}$ & $\cos \phi$ & $\epsilon^{\prime}$ & $\cos \phi$ & $\epsilon^{\prime}$ & $\cos \phi$ \\
\hline 0.75 & 25.80 & 0.784 & & 0.0495 & 22. & 0.787 & 13. 90 & \\
\hline 1.5 & 19 & 705 & & 0 & 17.70 & .715 & 11. 54 & .514 \\
\hline 3. 0 & 15 & 6 & & .0559 & 14. & & & 419 \\
\hline 6.0 & $1:$ & 5 & 4. 46 & .0649 & 11. & .5 & 8. & .325 \\
\hline 12.0 & 11. 8 & 40 & 4.3 & .0894 & 10. & 4 & 7. & .254 \\
\hline 24. & 9.3 & .34 & 4. 1 & .08 & 8. & 3 & 7. & 1 \\
\hline 48. & 8.4 & .27 & 3.9 & 10 & 8. & .26 & 6. & .149 \\
\hline 96.0 & 7. 96 & 198 & 3. 76 & .1057 & 7. 58 & .188 & 6.67 & .115 \\
\hline
\end{tabular}

TABLE 7. Effect of temperature and moisture on apparent dielectric constant and power factor of vegetable leather

\begin{tabular}{|c|c|c|c|c|c|c|c|c|}
\hline \multirow{3}{*}{$\begin{array}{c}\text { Fre- } \\
\text { quency }\end{array}$} & \multicolumn{8}{|c|}{ Dry } \\
\hline & \multicolumn{2}{|c|}{$23^{\circ} \mathrm{C}$} & \multicolumn{2}{|c|}{$-68^{\circ} \mathrm{C}$} & \multicolumn{2}{|c|}{$23^{\circ} \mathrm{C}$} & \multicolumn{2}{|c|}{$2^{\circ} \mathrm{C}$} \\
\hline & $\epsilon^{\prime}$ & $\cos \phi$ & $\epsilon^{\prime}$ & $\cos \phi$ & $\epsilon^{\prime}$ & $\cos \phi$ & $\epsilon^{\prime}$ & $\cos \phi$ \\
\hline $\begin{array}{l}k c \\
0.75 \\
1.5 \\
3.0 \\
6.0 \\
12.0 \\
24.0 \\
48.0 \\
96.0\end{array}$ & $\begin{array}{l}2.41 \\
2.39 \\
2.38 \\
2.36 \\
2.34 \\
2.33 \\
2.31 \\
2.30\end{array}$ & $\begin{array}{r}0.0199 \\
.0173 \\
.0163 \\
.0159 \\
.0152 \\
.0149 \\
.0143 \\
.0129\end{array}$ & $\begin{array}{l}2.14 \\
2.14 \\
2.13 \\
2.13 \\
2.12 \\
2.12 \\
2.11 \\
2.10\end{array}$ & $\begin{array}{r}0.00712 \\
.00832 \\
.00730 \\
.00699 \\
.00676 \\
.00638 \\
.00639 \\
.00592\end{array}$ & $\begin{array}{l}2.41 \\
2.39 \\
2.38 \\
2.37 \\
2.35 \\
2.33 \\
2.32 \\
2.30\end{array}$ & $\begin{array}{r}0.0191 \\
.0186 \\
.0163 \\
.0161 \\
.0154 \\
.0149 \\
.0144 \\
.0130\end{array}$ & $\begin{array}{l}2.31 \\
2.31 \\
2.30 \\
2.28 \\
2.27 \\
2.25 \\
2.24 \\
2.23\end{array}$ & $\begin{array}{r}0.0195 \\
.0171 \\
.0148 \\
.0139 \\
.0132 \\
.0126 \\
.0121 \\
.0108\end{array}$ \\
\hline \multirow{3}{*}{$\begin{array}{l}48.0 \\
96.0\end{array}$} & \multicolumn{8}{|c|}{$11.6 \% \mathrm{H}_{2} \mathrm{O}$} \\
\hline & \multicolumn{2}{|c|}{$23^{\circ} \mathrm{C}$} & \multicolumn{2}{|c|}{$-71^{\circ} \mathrm{C}$} & \multicolumn{2}{|c|}{$18^{\circ} \mathrm{C}$} & \multicolumn{2}{|c|}{$0^{\circ} \mathrm{C}$} \\
\hline & $\epsilon^{\prime}$ & $\cos \phi$ & $\epsilon^{\prime}$ & $\cos \phi$ & $\epsilon^{\prime}$ & $\cos \phi$ & $\epsilon^{\prime}$ & $\cos \phi$ \\
\hline $\begin{array}{r}0.75 \\
1.5 \\
3.0 \\
6.0 \\
12.0 \\
24.0 \\
48.0 \\
96.0\end{array}$ & $\begin{array}{l}\text { 3. } 56 \\
3.49 \\
3.43 \\
3.38 \\
3.34 \\
3.31 \\
3.27 \\
3.23\end{array}$ & $\begin{array}{r}0.0755 \\
.0564 \\
.0429 \\
.0359 \\
.0307 \\
.0275 \\
.0267 \\
.0237\end{array}$ & $\begin{array}{l}2.55 \\
2.52 \\
2.49 \\
2.46 \\
2.43 \\
2.40 \\
2.37 \\
2.32\end{array}$ & $\begin{array}{r}0.0319 \\
.0279 \\
.0273 \\
.0267 \\
.0268 \\
.0255 \\
.0254 \\
.0236\end{array}$ & $\begin{array}{l}3.70 \\
3.63 \\
3.57 \\
3.52 \\
3.49 \\
3.44 \\
3.41 \\
3.37\end{array}$ & $\begin{array}{r}0.0660 \\
.0498 \\
.0383 \\
.0333 \\
.0298 \\
.0276 \\
.0274 \\
.0261\end{array}$ & $\begin{array}{l}3.40 \\
3.37 \\
3.34 \\
3.30 \\
3.26 \\
3.23 \\
3.20 \\
3.16\end{array}$ & $\begin{array}{r}0.0377 \\
.0301 \\
.0264 \\
.0256 \\
.0250 \\
.0249 \\
.0265 \\
.0262\end{array}$ \\
\hline \multirow{3}{*}{$\begin{array}{l}48.0 \\
96.0\end{array}$} & \multicolumn{8}{|c|}{$23.2 \% \mathrm{H}_{2} \mathrm{O}$} \\
\hline & \multicolumn{2}{|c|}{$22^{\circ} \mathrm{C}$} & \multicolumn{2}{|c|}{$-74^{\circ} \mathrm{C}$} & \multicolumn{2}{|c|}{$23^{\circ} \mathrm{C}$} & \multicolumn{2}{|c|}{$0^{\circ} \mathrm{C}$} \\
\hline & $\epsilon^{\prime}$ & $\cos \phi$ & $\epsilon^{\prime}$ & $\cos \phi$ & $\epsilon^{\prime}$ & $\cos \phi$ & $\epsilon^{\prime}$ & $\cos \phi$ \\
\hline $\begin{array}{r}0.75 \\
1.5 \\
3.0 \\
6.0 \\
12.0 \\
24.0 \\
48.0 \\
96.0 \\
160.0\end{array}$ & $\begin{array}{r}14.06 \\
10.35 \\
9.20 \\
7.65 \\
6.69 \\
6.06 \\
5.66 \\
5.42\end{array}$ & $\begin{array}{r}0.417 \\
.435 \\
.406 \\
.373 \\
.327 \\
.233 \\
.171 \\
.119\end{array}$ & $\begin{array}{l}3.68 \\
3.60 \\
3.47 \\
3.40 \\
3.29 \\
3.18 \\
3.07 \\
2.97 \\
2.90\end{array}$ & $\begin{array}{r}0.0572 \\
.0610 \\
.0644 \\
.0690 \\
.0729 \\
.0746 \\
.0764 \\
.0709 \\
.0680\end{array}$ & $\begin{array}{r}13.00 \\
10.27 \\
8.50 \\
7.29 \\
6.55 \\
6.06 \\
5.75 \\
5.55\end{array}$ & $\begin{array}{r}0.493 \\
.471 \\
.416 \\
.346 \\
.268 \\
.196 \\
.143 \\
.100\end{array}$ & $\begin{array}{l}9.25 \\
7.63 \\
6.74 \\
6.14 \\
5.76 \\
5.53 \\
5.35 \\
5.21\end{array}$ & $\begin{array}{c}0.427 \\
.369 \\
.292 \\
.223 \\
.162 \\
.117 \\
.0869 \\
.0645\end{array}$ \\
\hline
\end{tabular}




\section{Discussion}

\subsection{Electrical Properties of Collagen and Leather}

The data obtained here are quite similar to results that have been reported for other fibrous materials, some of which might not be expected to be electrically comparable. With due consideration to the frequencies involved, values of dielectric constants of leather and collagen fibers are of the same order as values of silk and wool [11] as previously noted, as well as values on cellulose [7] obtained by Dakin. Earlier values on cellulose [4, 9, 43] are slightly different but of the same general order. The higher values for vegetable leather reported here are to be expected, since the appreciable amount of incorporated vegetable tannin probably has a higher dielectric constant than collagen, as shown by measurements of Knoke [25]. The reported dielectric constant of dry gelatin, 2.68 at $512 \mathrm{kc}$ [13], appears to be less than might be expected in view of its being derived from collagen.

Resistivity data are likewise similar to results previously reported on cellulose products that have been extensively studied in view of their widespread electrical applications. However, results obtained here for lea ther exhibit no linear relationship between logarithm of resistance and any simple function of moisture content as has been reported for textiles and cotton $[34,36,46]$. This divergence may be associated with the greater adsorptive capacity of leather for moisture.

Apparant or bulk dielectric constants of moist leather have been reported by Compton [6], who used a bridge method at $1 \mathrm{kc}$. His experimental values are much larger than those reported here at comparable moisture contents. This fact, together with his extremely high values of dielectric constant $-\epsilon^{\prime}$ as high as 4980 , will be discussed later. Neither Compton nor Kremen [26, 27] investigated the effects of temperature or frequency on $\epsilon^{\prime}$ and neither studied power losses, although Compton concluded that resistance measurements made during his measurements showed such little concordance that their use did not seem justified.

The data obtained here are similar in many respects to previous reports of effects of adsorbed vapors on the electrical properties of other porous materials $[1,8,22,23,24,31,35,44]$. In view of the similarities in results and the characteristics of the materials, the interpretations of the present data will be patterned to some extent along the lines of the conclusions of previous workers $[8,12,13,22,34,35$, $38,43]$, who have emphasized the importance of considering interfacial polarization in electrical measurements on nonhomogeneous materials of this type.

Leather must possess a large adsorption surface area by virtue of its fibrous structure. This area has been calculated by Kanagy [20] from adsorption data. Since the fibers are composed of submicroscopic fibrils and moisture appears to penetrate the fibers, it would appear that the fibrillar surface area must play a role in these data. The filaments that make up the fibrils Imay also be involved, but quali- tatively the conclusions reached here will be independent of the units involved. There are probably three phases present in moist leather-the leather substrate, which may contain combined or dissolved moisture; a surface layer of adsorbed moisture; and the surrounding air. The interface between any two of these phases is an obvious source of MaxwellWagner polarization effects. The behavior of such types of interfacial polarization has been considered in detail and developed quantitatively for simple systems by other workers [32, 38, 39]. In leather the vast number of these interfaces present, the complexity of the interconnections of the physical matrix, and the unknown conductivities and dielectric constants of the phases involved present a problem that permits no quantitative analysis. The only facts available are that the dielectric constants of the three phases are quite different in bulk form, while the conductivity of the air will be much less than that of either the moist substrate or the adsorbed moisture layer. Wagner [39] has shown that a necessary condition for minimizing interfacial effects is that

$$
\sigma_{1} \epsilon_{1}^{\prime}=\sigma_{2} \epsilon_{2}^{\prime}
$$

where $\sigma$ and $\epsilon^{\prime}$ are conductivity and dielectric constant, respectively, and the subscripts refer to the phases that form the interface. In the present situation it is extremely improbable that such a relationship will hold at the three possible interfaces at all moisture contents studied.

A somewhat different type of polarization, known as electrode polarization, may exist at the discontinuity between specimens and the condenser plates. In the present experiments no differentiation between electrode polarization and the interfacial polarization existing within the specimen is possible, and the electrode polarization will be considered as lumped with the interfacial effects.

The other important type of polarization arises as a result of the numerous permanent dipoles present in the basic protein by virtue of its amino acid composition. These dipoles will represent sources of Debye polarization, an effect that is qualitatively similar to interfacial polarization. The extreme difficulty in distinguishing between these two phenomena has been emphasized previously [32, 38].

The qualitative interpretation of the data in tables 2 to 7 appears to require at least two polarization processes, one being freely operative only at very low frequencies at high moisture contents and in all probability associated with interfacial effects, the other being effective up to much higher frequencies and probably arising from dipolar effects. The relative importance of these two effects is determined by the moisture content of the specimen.

The strong absorption region at low frequencies predominates at moisture contents above approximately 15 percent, except at low temperatures. This dielectric absorption is attributed to interfacial polarization phenomena. The rapid decrease of $\epsilon^{\prime}$ and $\cos \phi$ with increasing frequency and decreasing temperature may be logically attributed to this 
effect. The dependence of capacitance and resistance on time at a constant frequency is suggestive of charging currents and therefore of polarization at a discontinuity. The data of Compton [6], who worked in this frequency range, can be interpreted to a large extent as arising from interfacial effects. The specimens used by Compton in all probability had a much lower resistivity than those prepared here. This increased conductivity permits a larger charging current and an apparent increase in dielectric constant [39]. Thus Compton's dielectric constants at comparable moisture contents and frequency vary from at least three to several hundred times the present values. The extremely high values of $\boldsymbol{\epsilon}^{\prime}$ reported by Compton- $\boldsymbol{\epsilon}^{\prime}$ as high as 4980 on chrome leather containing only 26 percent of moisture - can be logically interpreted only on the basis of interfacial effects arising from appreciable conductivity in the test specimens. For the foregoing reasons it is believed that at high moisture contents the present data are obtained on the descending slope of a large interfacial power absorption region that persists to very low frequencies.

At low moisture contents - that is, below approximately 15 percent of moisture-the electrical behavior is quite different. In this region it would appear that the data is obtained in a region between two dielectric absorption maxima, the interfacial region at low frequencies, which diminishes in importance with decreased moisture content, and a second dielectric absorption region with a maximum at high frequencies far beyond the experimental range of frequency. This second dielectric absorption maximum would account for the increased losses with increasing frequency observed in this moisture range, as well as the increase in loss observed on decreasing the temperature. This latter effect arises as a result of the analogy between frequency and temperature, a reduction in temperature being experimentally equivalent to increasing the frequency of the measurements or to shifting the dielectric relaxation effects to lower frequencies.

At high moisture contents at $-70^{\circ} \mathrm{C}$ the interfacial effects have virtually disappeared as a result of reduced conductivity at low temperatures [39], but a distinct dielectric absorption maximum in $\cos \phi$ appears in two specimens in the experimental frequency range - the corresponding maximum in $\epsilon^{\prime \prime}$ appears for all three specimens. This dielectric absorption maximum is not observed under similar conditions for dry leather or at low moisture contents, so that the moisture appears to be largely responsible for the observed effect. Since a dielectric absorption maximum has been indicated at high frequencies at room temperature, this maximum may have been shifted to the experimental frequency range by the temperature reduction. However, since a similar effect is not observed at lower moisture contents, it would be necessary to assume that the frequency of the absorption maximum is dependent on moisture content. It is also possible that this dielectric absorption maximum is attributable to ice, which is known to exhibit such a maximum in this frequency range, but at a somewhat higher temperature $[10$, $33,40]$.

As a result of the foregoing considerations, the following general picture may be given. In the dry condition in this frequency range, leather may be considered to have a dielectric behavior that is characterized by a moderate power absorption with slight frequency dependence. As the moisture content of the leather increases, two dielectric absorption maxima one at very high, the other at very low frequencies - appear and increase in magnitude with increasing moisture content. The high-frequency maximum grows the more rapidly at low moisture contents, and at very low moisture contents largely determines the behavior of the leather. As moisture is added the low frequency, or interfacial effect, grows at an increasing rate, and in the moisture range 5 to 15 percent, losses due to both types of dielectric absorption are determining factors. In this moisture range the data may be pictured as being taken in the trough between the two dielectric absorption maxima. As the moisture content rises above about 15 percent, the rate of growth of the interfacial region accelerates tremendously, and at high moisture contents the enormous size of the power absorption arising from interfacial effects completely overshadows the smaller but probably still important maximum at higher frequencies. The source of the low-frequency losses has been attributed to interfacial charging phenomena, and it remains to consider the causes of the losses at higher frequencies. The high-frequency absorption may be due to dipolar polarization or to a different type of interfacial phenomenon. Since dipoles are known to be present in the protein, it would seem that the power absorption may be due to such causes. The increasing importance of this absorption with increasing moisture content, noted at low moisture contents but probably occurring even at high moisture contents, may be considered as due to increased mobility of the existing dipoles conferred by the imbibed moisture. The dipoles in the dry material may be thought of as being largely immobilized by the compact molecular structure of dry collagen with correspondingly large restraining forces. Since the compact structure relaxes in the presence of moisture, these bound dipoles may be freed to some extent, the degree of freedom increasing with increasing moisture content.

The interpretation of these data given in the foregoing implies a somewhat different role of moisture in the low and high-moisture ranges, with a gradual shift of the effect centering at approximately 15 percent of moisture. This behavior is clearly shown by $\epsilon^{\prime}$ and $\cos \phi$ in the tabular data. The gradual transition region occurs at lower moisture contents in vegetable leather than in chrome leather or in hide, and may be directly related to that moisture content range in which the slope of the heat of adsorption curve is undergoing its greatest change [20]. The apparent difference in effects of moisture in the lowand high-moisture range may be considered to be due to the decreased binding energy of the moisture 
with increasing moisture content. Such concepts have been advanced previously $[1,35]$.

This difference in behavior of moisture in the two moisture ranges may be related to concepts of "bound water", which have been discussed by numerous workers $[2,15,16,18,19]$, and with particular reference to leather by Compton [6], Kremen [28], and Cheshire and Holmes [5]. These latter workers conclude that between 30 and 50 percent of moisture on a hide substance basis can be considered as bound water. The present studies were conducted principally in the range of moisture contents in which these workers would consider all moisture present to be bound.

The present data show that the moisture present in small amounts produces a markedly different effect than that present in large quantities. Below a rather ill-defined transition region centering at about 15 percent of moisture, the principal effect is the enhancement of the high-frequency dielectric absorption, while interfacial polarization is effected to a rather small degree. In this range the moisture might be considered to be mainly intimately associated with the protein, probably by means of chemical forces through hydrogen bonding. Relatively little moisture is therefore available to act electrically as one might expect water to behave.

At the higher moisture contents the effect of moisture on the high-frequency dielectric absorption is masked by the rapid growth of the interfacial polarization. In this range the principal effect appears to be on the surface of the structural units involved, so that conducting layers are rapidly built up to produce the interfacial effects observed. In this range the moisture produces electrical results that might be expected of water.

It is possible, therefore, to distinguish between these two essentially different roles of moisture, even in the range in which all moisture has been considered to be bound. It must be concluded that there is considerable difference in binding of the so-called bound water.

\section{Determination of Moisture in Leather by Electrical Methods}

From the data obtained in these studies it follows that in this frequency range electrical methods cannot be expected to yield precise results in quantitative analyses for moisture. To test this conclusion an experiment was performed on similar specimens of commercial, fat-liquored, chrome upper leather that were cut from corresponding areas of different hides of the same tannage. All specimens were cut from similar positions in the shoulder region, and were selected for uniformity of thickness over the area of the test piece. Following initial measurements, the specimens were degreased and rerun to ascertain the effects of inequalities in grease content. The results of these measurements are given in table 8 .

Thickness measurements are the average of 15 determinations distributed uniformly over the area of the test specimen. These measurements were
TABLE 8. Reproducibility of electrical data on specimens from different hides of the same tannage ${ }^{\text {s }}$

$\sigma$ for $\epsilon^{\prime}$ commercial $=0.10 ; \sigma$ for $\epsilon^{\prime}$ degreased $=0.13$

\begin{tabular}{|c|c|c|c|c|c|c|c|c|}
\hline \multirow[b]{2}{*}{$\begin{array}{l}\text { Spec- } \\
\text { imen }\end{array}$} & \multicolumn{4}{|c|}{ Commercial leather } & \multicolumn{4}{|c|}{ Degreased leather } \\
\hline & $\begin{array}{l}\text { Thick- } \\
\text { ness }\end{array}$ & $\begin{array}{l}\text { Condens- } \\
\text { er plate } \\
\text { separa- } \\
\text { tion }\end{array}$ & $\epsilon^{\prime}$ & $\cos \phi$ & $\begin{array}{c}\text { Thick- } \\
\text { ness }\end{array}$ & $\begin{array}{l}\text { Condens- } \\
\text { er plate } \\
\text { separa- } \\
\text { tion }\end{array}$ & $\epsilon^{\prime}$ & $\cos \phi$ \\
\hline $\begin{array}{l}1 \\
2 \\
3 \\
4 \\
5\end{array}$ & $\begin{array}{c}\text { in. } \\
0.093 \\
.094 \\
.094 \\
.090 \\
.094\end{array}$ & $\begin{array}{l}\text { in.b } \\
1.1057 \\
1.1040 \\
1.1059 \\
1.1078 \\
1.1060\end{array}$ & $\begin{array}{r}3.29 \\
3.35 \\
3.43 \\
3.18 \\
3.42\end{array}$ & $\begin{array}{r}0.0275 \\
.0356 \\
.0382 \\
.0269 \\
.0444\end{array}$ & $\begin{array}{r}\text { in. } \\
0.094 \\
.094 \\
.094 \\
.092 \\
.095\end{array}$ & $\begin{array}{c}\text { in.b } \\
1.1079 \\
1.1071 \\
1.1075 \\
1.1037 \\
1.1087\end{array}$ & $\begin{array}{l}3.26 \\
3.18 \\
3.41 \\
3.10 \\
3.38\end{array}$ & $\begin{array}{r}0.0521 \\
.0485 \\
.0805 \\
.0448 \\
.0599\end{array}$ \\
\hline
\end{tabular}

- Measurements at $3 \mathrm{kc}$.

b Measured from arbitrary zero.

made to the nearest $0.001 \mathrm{in}$. Condenser plate separations are the average of three measurements made as described previously, and are accurate to the nearest 0.0001 in. Differences noted in $\epsilon^{\prime}$ a od $\cos \phi$ are real, since the experimental capacitances and resistances involved were measured to at least four significant figures.

Variations noted in $\epsilon^{\prime}$ and $\cos \phi$ indicate that use of these quantities to characterize or measure the moisture content would be subject to considerable uncertainty in an arbitrary piece of leather. Measurements of moisture contents of these specimens have not been made, sinco all specimens were subjected to identical treatments throughout, and experience in this laboratory has shown that a moisture content of approximately 12 percent with a variation of less than approximately 1 percent may be expected for such specimens from the same location of different hides of the same tannage. Since some differences in apparent density or fiber orientation exist, the variations observed in $\epsilon^{\prime}$ and $\cos \phi$ are not considered excessive.

It is concluded, therefore, that in this frequency range electrical methods are not expected to produce precise quantitative determinations of moisture in leather. Although this conclusion is reached largely as a result of data accumulated using alternating currents, it is believed that the same considerations apply to direct-current measurements. This extension of the conclusions appears to be valid as a result of observations made previously concerning behavior of the direct-current resistance of specimens containing moderate amounts of moisture. For rapid, nondestructive estimates of moisture content, particularly for control work, such methods may be adequate, provided the effects of temperature and salt content are recognized and controlled.

The author is indebted to C. G. Malmberg and A. A. Maryott for use of the capacitance bridge that made these measurements possible, as well as for the stimulating discussions directed toward this problem.

\section{References}

[1] G. Argue and O. Maass, Can. J. Research 13B, 156 (1935).

[2] D. R. Briggs, J. Phys. Chem. 36, 367 (1932).

[3] 'E. F. Burton and Arnold Pitt, Can. J. Research 1, 155 (1929). 
[4] A. Campbell, Proc. Roy. Soc. (London) 78A, 196 (1907).

[5] A. Cheshire and N. L. Holmes, J. Int. Soc. Leather Trades Chem. 26, 237 (1942).

[6] E. D. Compton, J. Am. Leather Chem. Assoc. 39, 74 (1944).

[7] T. W. Dakin, Annual Report 1950; Conference on Electrical Insulation Nat. Acad. Sci., Nat. Research Council (March 15, 1951).

[8] T. W. Dakin and R. W. Auxier, Ind. Eng. Chem. 37, 268 (1945).

[9] H. A. DeLuca and W. B. Campbell, Can. J. Research 16, $273(1938)$

[10] M. J. Errera, J. Phys. Radium 5, 304 (1924).

[11] M. J. Errera and Sack, Ind. Eng. Chem. 35, 712 (1943)

[12] H. Fricke and H. J. Curtis, J. Phys. Chem. 41, 729 1937).

[13] H. Fricke and E. Parker, J. Phys. Chem. 44, 716 (1940).

[14] S. D. Gardiner, J. Soc. Chem. Ind. 62, 75 (1943).

[15] R. A. Gortner, Outlines of biochemistry (John Wiley \& Sons, Inc., New York, N. Y., 1938).

[16] W. F. Hampton and J. H. Mennie, Can. J. Research 10, 452 (1934).

[17] Handbook of chemistry and physics, 30 ed. (Chemical Rubber Publishing Co., Cleveland, Ohio).

[18] W. R. Horn and J. H. Mennie, Can. J. Research 12, 702 (1935).

[19] I. D. Jones and R. A. Gortner, J. Phys. Chem. 36, 387 (1932).

[20] J. R. Kanagy, J. Research NBS 44, 31 (1950) RP2056.

[21] J. R. Kanagy, J. Research NBS 38, 119 (1947) RP1763.

[22] G. King, Trans. Faraday Soc. 43, 601 (1947).

[23] G. King, Nature 158, 134 (1946).

[24] G. T. Kohman, Ind. Eng. Chem. 31, 807 (1939).

[25] S. Knoke, Z. Elektrochem. 43, 749 (1937).

[26] S. S. Kremen, Dissertation, University of Cincinnati (1949).
]27] S. S. Kremen, J. Am, Leather Chem, Assoc, 44, 774 (1949).

[28] S. S. Kremen and R. M. Lollar, J. Am. Leather Chem. Assoc. 46, 34 (1951).

[29] C. G. Malmberg and A. A. Maryott, J. Research NBS 45, 299 (1950) RP2137.

[30] R. H. Marriott, J. Int. Soc. Leather Trades Chem. 19, 133 (1935)

[31] R. MeIntosh, H. S. Johnson, N. Hollies, and L. McCleod, Can. J. Research 25B, 566 (1947).

[32] S. O. Morgan, Trans. Electrochem. Soc. 65, 109 (1934).

[33] E. J. Murphy, Trans. Electrochem. Soc. 65, 133 (1934).

[34] E. J. Murphy, J. Phys. Chem. 33, 200 (1929).

[35] E. J. Murphy and H. H. Lowry, J. Phys. Chem. 34, 598 (1930).

[36] E. J. Murphy and A. C. Walker, J. Phys. Chem. 32, 1761 (1928).

[37] S. B. Newman, private communication.

[38] H. H. Race, Trans. Am. Inst. Elec. Engrs. 52, 682 (1933).

[39] H. Schering, Die Isolierstoffe der Electrotechnik, chapt. I by K. W. Wagner (Julius Springer, Berlin, 1924).

[40] C. P. Smyth and C. S. Hitchcock, J. Am. Chem. Soc. 54, 4631 (1932).

[41] J. L. Spencer-Smith, J. Textile Inst. 26, T336 (1935).

[42] H. Starke, Verhandl. deut. physik Ges. 15, 69 (1896).

[43] W. N. Stoops, J. Am. Chem. Soc. 56, 1480 (1934).

[44] J. Tausz and H. Rumm, Kolloid-Beihefte 39, 58 (1934).

[45] H. Vollmar, Z. Elektrochem. 50, 150 (1944).

[46] A. C. Walker, J. Text. Inst. 24, T145 (1933).

[47] C. E. Weir, J. Research NBS 41, 279 (1948) RP1924.

Washington, December 13, 1951. 